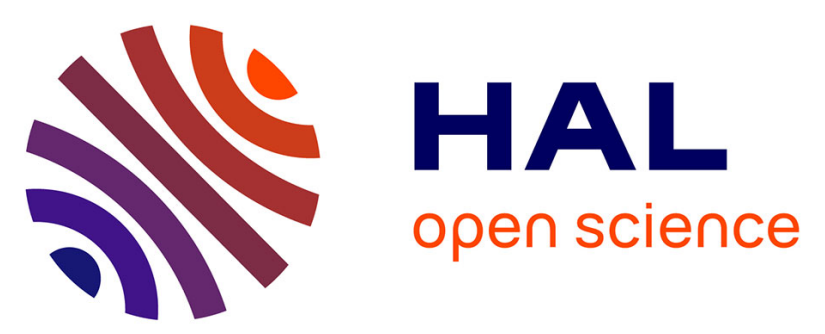

\title{
Time-resolved optical fluorescence spectroscopy of heterogeneous turbid media with special emphasis on brain tissue structures including diseased regions: $A$ sensitivity analysis
}

Fabrice Vaudelle, Jean-Pierre L'Huillier

\section{To cite this version:}

Fabrice Vaudelle, Jean-Pierre L'Huillier. Time-resolved optical fluorescence spectroscopy of heterogeneous turbid media with special emphasis on brain tissue structures including diseased regions: A sensitivity analysis. Optics Communications, 2013, 304, pp.161-168. 10.1016/j.optcom.2013.04.016 . hal-01063761

\section{HAL Id: hal-01063761 \\ https://hal.science/hal-01063761}

Submitted on 12 Sep 2014

HAL is a multi-disciplinary open access archive for the deposit and dissemination of scientific research documents, whether they are published or not. The documents may come from teaching and research institutions in France or abroad, or from public or private research centers.
L'archive ouverte pluridisciplinaire HAL, est destinée au dépôt et à la diffusion de documents scientifiques de niveau recherche, publiés ou non, émanant des établissements d'enseignement et de recherche français ou étrangers, des laboratoires publics ou privés. 


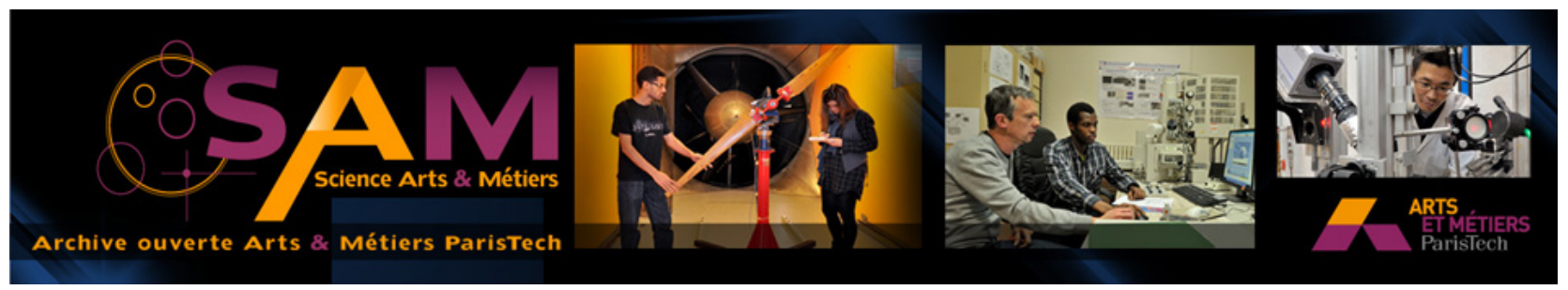

\section{Science Arts \& Métiers (SAM)}

is an open access repository that collects the work of Arts et Métiers ParisTech researchers and makes it freely available over the web where possible.

This is an author-deposited version published in: http://sam.ensam.eu

Handle ID: .http://hdl.handle.net/10985/8511

\section{To cite this version :}

Fabrice VAUDELLE, Jean-Pierre L'HUILLIER - Time-resolved optical fluorescence spectroscopy of heterogeneous turbid media with special emphasis on brain tissue structures including diseased regions: A sensitivity analysis - Optics Communications - Vol. 304, p.161-168 - 2013 


\title{
Time-resolved optical fluorescence spectroscopy of heterogeneous turbid media with special emphasis on brain tissue structures including diseased regions: A sensitivity analysis
}

\author{
Fabrice Vaudelle*, Jean-Pierre L'huillier \\ Arts et Métiers ParisTech, LAMPA, Equipe ECPS, 2 Boulevard du Ronceray, Angers 49035, France
}

Keywords:

Fluorescence

Brain tissue model

Inclusion

Time-resolved spectroscopy

Monte Carlo simulations

\begin{abstract}
A B S T R A C T
Fluorescence-enhanced optical imaging based on near-infrared light provides a promising tool to differentiate diseased lesions from normal tissue. However, the measurement sensitivity of the fluorescence signals acquired at the output surface of the tissue is greatly influenced by the tissue structure, the optical properties, the location and the size of the target. In this paper, we present a numerical model based on the Monte Carlo method that allows to simulate time-resolved reflectance signals acquired on the surface of the scalp of a human head model bearing a fluorescent diseased region (tumor, glioma). The influence of tumor depth, tumor size and tumor shape evolution on the computed signals are analyzed by taking into account the multi-layered tissue structure. The simulations show that the mean-time-of-flight and the difference between two mean-times acquired at two source-detector distances are both relevant to this problem type. Furthermore, the simulations suggest that the use of the difference between mean-flight-times may be interesting to probe scattering changes that occur in the cerebrospinal fluid (CSF).
\end{abstract}

\section{Introduction}

Investigation of complex interactions between light and biological material is of great interest since optical methods provide a noninvasive way of assessing and imaging the optical properties of the tissue. Endogenous molecules of most biological tissue have specific absorption spectra and then can selectively be excited to deliver information related to the pathology of these structures. Such investigations are, however, limited to the subsurface of the interrogated organs owing to the small quantum yields of the intrinsic chromophores and the wavelength range (UV) of the excitation light [1,2]. Intrinsic optical contrast based on the use of the near-infrared light $(650-900 \mathrm{~nm})$, allows to diagnose more deeply into the tissue [3], but suffers from insufficient sensitivity [4,5] for localizing small $(<10 \mathrm{~mm})$ vascularized tumors. This situation might be improved by using exogenous contrast agents which accumulate at the tumor site. When excited by laser light, these contrast agents emit light at larger wavelengths (Stokes shift), thus allowing to analyze other several properties such as concentration, quantum yields, and mean lifetime [6,7]. Extensive research is being carried out to develop biomarkers which emit in

\footnotetext{
* Corresponding author. Tel.:+33 241207385.

E-mail addresses: f.vaudelle@libertysurf.fr (F. Vaudelle). jean-pierre.lhuillier@angers.ensam.fr (J.-P. L'huillier).
}

the near-infrared region. This has the main advantage of recording fluorescence signals issued from deep structures, typically in the order of several centimeters [8], and to reduce the auto fluorescence of the tissue [9]. However, scattering limits spatial resolution and contributes to blur the recorded (or reconstructed) images. Therefore, the diffusive optical imaging requires an interpretation on measurements performed from sources and detectors spaced over the boundaries of the interrogated tissue. In this way, relevant parameters linked to the localization of fluorescent inclusions in homogeneous media have been reported by different groups [10-19]. Optimizing analysis of these types of data is an area of very active research in order to improve depth sensitivity $[20-24]$ and quantify fluorescence rate $[25,26]$ in the human brain. Classically, noninvasive optical brain imaging may use continuous wave (CW), frequency-domain (FD), and time-domain (TD) instrumentations [27]. It was suggested, however, that time-resolved arrangement can be the best method for obtaining deep light penetration in the head from measurements made at longer delay times [20] and for assessing of brain hemodynamics [28]. The use of specific markers is now an opportunity to enhance the contrast between glioblastomas and healthy brain tissue in preclinical animal models $[29,30]$. The translation in human clinical setting needs great work to reveal the sensitivity of this diagnostic method as a function of target depth, target growing, and complex internal boundaries of the head tissue. Different human head models that include two-layer slab $[31,32]$, three- and five-layer 
structures [21,23] or MRI segmented slices [20,22-24] have been used by several groups. The presence of a relatively clear layer (cerebrospinal fluid-CSF), which surrounds the brain and fills the ventricular system, has been shown especially to alter the propagation of light in the head $[21,23,33]$. To account for the effect of low scattering property of the CSF which cannot be well portrayed by the diffusion approximation, adequate computational methods are needed. Monte Carlo codes $[20,22,34,35]$ and the hybrid Monte Carlo-diffusion method [23] have previously proved their efficiency to perform photon migration simulations in human head models including non-scattering CSF layer. To our knowledge, limited prior studies have investigated the use of time-resolved method to localize fluorescent inclusions embedded in complex brain tissue structures, and to explore the optical changes which can occur in the cerebrospinal fluid (CSF).

This paper illustrates the use of a Monte Carlo algorithm for investigating questions related to the detection of various fluorescent inclusions which are representative of disordered regions (tumors, glioblastomas) in brain tissue, and to optical changes in the CSF layer. A primary goal was to perform numerical simulations that show how relevant time parameters can serve as indicators on depth, size and shape of the inclusion, and provides information on possible density changes in the CSF layer.

\section{Methods}

\subsection{Brain tissue model}

Rather than being a simple tissue layer, the CSF depicts an irregular shape that varies between the sulci and gyri of the brain (Fig. 1(a)). Nevertheless, a pioneering work [21] showed that the geometries of the sulci and the boundary between the gray matter and the white matter scarcely affect the optical path of the photons. Furthermore, working with low source-detector separations $(\sim 1 \mathrm{~cm})$ suggests to neglect the effect of the regional scalp curvature. These arguments lend credit to the idea of simplifying the head structure as a semi-infinite multi-layer geometry.

To study the possibility to localize a fluorescent object embedded in an adult head model and investigate the effect of changes in scattering amount of CSF layer, we then used a simple adult head model that consists of five different plane homogeneous media including a surface layer (scalp and skull), a CSF layer, a gray matter layer and a white matter layer (see Fig. 1(b)). The thickness of each layer was varied according to different simulations reported below, whereas the assigned optical properties have been chosen from the data reported by the literature. To reduce notably the MC calculation time [20,34], the anisotropy coefficient $g$ was set to a low value equal to 0.1 for all tissue types, except for the CSF layer (thickness $<4 \mathrm{~mm}$ ) for which $g$ was fixed at 0.9 . The multi-layered structure comprises a fluorescent object located at the depth $z_{\mathrm{s}}$ from the surface of the scalp. Unless otherwise specified in the text, this object is assumed as having a spherical shape of radius $r$ varying from 2 to $12 \mathrm{~mm}$, and the source location was fixed and taken to be the origin of the coordinate system. This object modeled a native disordered region in the brain (tumor, glioblastoma) tagged by a fluorescent marker with a fixed uniform concentration $C(\mu \mathrm{M})$. The used marker had an extinction coefficient $\varepsilon=10^{4} \mathrm{M}^{-1} \mathrm{~mm}^{-1}$, a coefficient of absorption $\mu_{\mathrm{af}}=2.3 \varepsilon \mathrm{C}$ $\left(\mathrm{mm}^{-1}\right)$, a quantum efficiency $\eta=0.016$, and a fluorescence lifetime $\tau=0.56 \mathrm{~ns}$, with absorption/emission peaks at $780 \mathrm{~nm} / 830 \mathrm{~nm}$. These optical parameters are close to those of the Indocyanine Green (ICG), which is a nonspecific marker used routinely during ophthalmology procedures [36], but also for brain perfusion quantitation [25,26]. According to Eggert and Blazek [37], the optical properties of the inclusion (without addition of ICG) were chosen as similar to those of the gray matter layer (see Section 3.2). All the parameters used in the simulations are listed in Table 1.

\subsection{Monte Carlo fluorescence modeling}

All the simulations related to fluorescence re-emission were carried out by means of a Monte Carlo code mainly described and implemented by Wang et al. [38]. The approach towards modifying the model involves the consideration of a fluorescence algorithm which is able to simulate time-domain fluorescence photon generation and random walk of the individual photons through the multi-layered tissue structure.

Basically, a collimated beam impinging normally to the braintissue model is simulated by choosing the initial direction downward into the tissue. Each incident photon comprising a packet of photons and launched at the surface is considered as an excitation photon with a weight $(W)$ sets to unity. This weight is attenuated after each step to account for absorption losses in the medium following Beer's law. After each scattering step, a new direction for the photon path is decided according to a scattering phase function as given by Henyey-Greenstein. The flight of the photon is terminated (or the photon packet) when it crosses the top layer and is recorded as reflectance, or when its weight falls below a previously specified weight. Therefore, the photon weight is set to zero based on the Russian roulette technique, this also ensure the energy conservation. The time $t$, spent by the photon at the end of its travel through the medium is calculated as $t=n L / c_{0}$, where $L$ is the total path-length of the photon, $c_{0}$ the velocity of light in the vacuum, and $n$ is the refractive index of each layer composing the medium $(n=1.4)$. The localization of the fluorescent source point is obtained by means of an additional Russian roulette based on a probability of absorption $P$ [35]. This conversion probability of absorption of an excitation photon between two consecutive events was calculated along the path-length $l_{\mathrm{j}}$ inside the region a

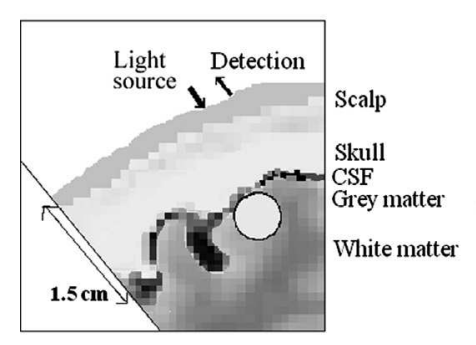

b

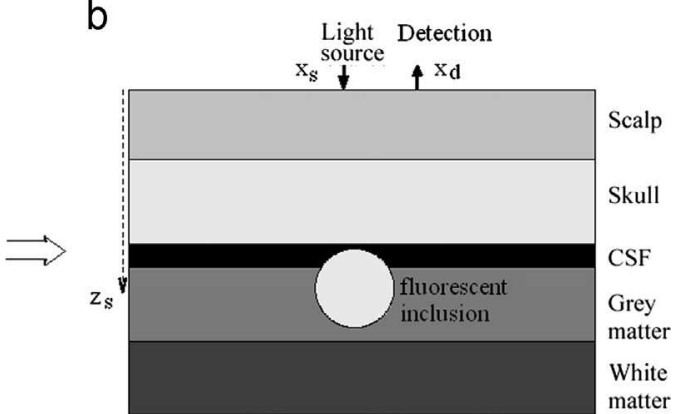

Fig. 1. (a) Realistic slice of an adult head model showing the complex boundaries between the main tissue components: scalp, skull, CSF layer, gray matter and white matter. (b) Schematic design of a five-layered adult head model bearing a spherical fluorescent object that mimics a diseased region of radius $r$, and located at the depth $z_{\mathrm{s}}$. 
Table 1

Optical properties of the tissues composing the adult head model as displayed in Fig. 1(b).

\begin{tabular}{lllc}
\hline Tissue type & Thickness & $\mu_{a}\left(\mathrm{~mm}^{-1}\right)$ & $\mu_{s}^{\prime}\left(\mathrm{mm}^{-1}\right)$ \\
\hline Scalp & $0,3-4 \mathrm{~mm}$ & $0.018^{\mathrm{a}}$ & $1.27,1.71,1.9^{\mathrm{a}}, 1.4$ \\
Skull & $4-7 \mathrm{~mm}$ & $0.016^{\mathrm{a}}, 0.04^{\mathrm{b}}$ & $1.07,1.44,1.6^{\mathrm{a}}$ \\
CSF & $1-4 \mathrm{~mm}$ & $0.004^{\mathrm{a}}, 0.04$ & 0.1 \\
Gray matter & $4-8 \mathrm{~mm}$ or infinity & $0.036^{\mathrm{a}}, 0.025^{\mathrm{b}}$ & $0.1,0.25^{\mathrm{a}}, 1,1.6$ \\
White matter & 0, infinity & $0.014^{\mathrm{a}}$ & $1.25^{\mathrm{b}}, 2.2^{\mathrm{a}}$ \\
\hline
\end{tabular}

a Okada et al. [21] $\lambda \sim 800 \mathrm{~nm}$.

${ }^{\mathrm{b}}$ Boas et al. [20] $\lambda>800 \mathrm{~nm}$.

tagged by the fluorophores, by the relationship

$P=\eta\left[1-\exp \left(-\Sigma j \mu_{a f} l_{j}\right)\right]$

Unlike the intrinsic fluorescence in layered tissue [39], we have bounded the region where the conversion of frequency will occur and we have just looked for the positions (cylindrical coordinates $\left.r_{\mathrm{f}}, \theta_{\mathrm{fi}}, z_{\mathrm{fi}}\right)$ of excitation inside a small volume and the time delay $t_{i}^{(0)}$ to reach them. However, to accelerate the Monte Carlo code, an approach that applies convolution and forward emission was used [39]. We have not considered a single conversion related to a point source $\left(r_{\mathrm{fi}}, \theta_{\mathrm{f}}, z_{\mathrm{fi}}\right)$, but several, in order to have a whole series of possible optical lengths $\left(l_{k, i}^{(F)}=c_{0}\left[t-t_{1}^{(0)}\right]_{k} / n\right)$. Then, newly created fluorescence photons continue to propagate according to the same weighted photon model, albeit using specific optical properties for the emission wavelength. The signal acquired at the abscissa $x_{\mathrm{d}}$ was then obtained by summing fluorescent photons coming from all the optical paths $l_{\mathrm{k}, 1}^{(F)}$. The finite fluorescence lifetime $\tau$ was also taken into account by convolution of the recorded time-dependent data with the decay rate of the marker $(1 / \tau) \exp (-t / \tau)$, as reported in $[35,39-41]$.

Next, the code allows to computing different time relevant parameters from the consideration of the temporal reflectance data $[10,12,15,40-43]$ : time to reach the half of the maximum fluorescence intensity $t_{1 / 2}$; -the first moment or the mean-time-ofphoton-flight defined as

$\langle t\rangle=\frac{\int_{0}^{\infty} t F(t) \mathrm{d} t}{\int_{0}^{\infty} F(t) \mathrm{d} t}$

and -the difference between two mean times $\Delta\langle t\rangle=\left\langle t x_{\mathrm{di}+1}\right\rangle-\left\langle t x_{\mathrm{di}}\right\rangle$ acquired at two source-detector distances $x_{\mathrm{di}+1}$ and $x_{\mathrm{di}}$.

\section{Results and discussion}

Two series of investigations were performed in this paper. The former is related to simulations on homogeneous tissue with various source-detector separations, radii, concentrations of ICG, and depths of the fluorescent object. This allows to compare Monte Carlo results with those obtained from analytical model [11] and recent experiments [32], and also exemplify the interest of using the three relevant time parameters $t_{1 / 2},\langle t\rangle$ and $\Delta\langle t\rangle$ in these problem types. The second series is concerned with the imaging of a three- or a five-layer brain tissue model, including controlled shape of the excited fluorophores, thickness and optical properties of CSF layer. This provides information on possible physiological changes that occur in diseased human head.

In these instances, the different simulations ignore the residual background fluorescence, and then assume that all the fluorescence arises from the object located either inside the homogeneous tissues or in the multi-layered tissue structures, as is the case for perfect uptake scenario.

\subsection{Homogeneous media}

Fig. 2(a) shows the evolution of both $t_{1 / 2}$ and $\langle t\rangle$ as a function of the depth location $z_{\mathrm{s}}$ of a fluorescent object $(r=2 \mathrm{~mm})$ containing $1 \mu \mathrm{M}$ of ICG $\left(\mu_{\mathrm{af}}=0.023 \mathrm{~mm}^{-1}\right)$ and immersed in a homogeneous medium with the optical properties $\mu_{a}=0.03 \mathrm{~mm}^{-1}$ and $\mu_{\mathrm{s}}{ }^{\prime}=$ $1.5 \mathrm{~mm}^{-1}$. All the simulations refer to three different sourcedetector separations, $x_{\mathrm{d}}=3,5$ and $8 \mathrm{~mm}$. Monte Carlo code data were compared to the results achieved with a simplified analytical model reported by Hattery et al. [11], where the time-dependent flux is defined before the convolution with the decay rate of the fluorescent marker, as:

$$
\begin{aligned}
& \sum_{\varepsilon_{1}, \varepsilon_{2}} \varepsilon_{1} \varepsilon_{2} h\left(\frac{3 \mu_{\mathrm{s}}^{\prime}}{4}\left[x_{\mathrm{s}}^{2}+y_{\mathrm{s}}^{2}+\left(z_{\mathrm{s}}+\varepsilon_{1} \frac{\sqrt{2}}{\mu_{\mathrm{s}}^{\prime}}\right)^{2}\right],\right. \\
& \left.\frac{3 \mu_{\mathrm{s}}^{\prime}}{4}\left[\left(x_{\mathrm{s}}-x_{\mathrm{d}}\right)^{2}+\left(y_{\mathrm{s}}-y_{\mathrm{d}}\right)^{2}+\left(z_{\mathrm{s}}+\frac{\sqrt{2}}{\mu_{\mathrm{s}}^{\prime}}+\varepsilon_{2} \frac{\sqrt{2}}{\mu_{\mathrm{s}}^{\prime}}\right)^{2}\right]\right)
\end{aligned}
$$

where

$h(\alpha, \beta)=\frac{\sqrt{\alpha}+\sqrt{\beta}}{\left(c t \mu_{\mathrm{s}}^{\prime}\right)^{3 / 2}(\pi \alpha \beta)^{1 / 2}} \exp \left(\frac{\sqrt{\alpha}+\sqrt{\beta}}{c t \mu_{\mathrm{s}}^{\prime}}-c \mu_{\mathrm{a}} t\right)$

with

$\varepsilon_{i}= \pm 1, \quad c=c_{0} / n$

As displayed Fig. 2(a), we note that $t_{1 / 2}$ and $\langle t\rangle$ are both sensitive to the inclusion depth and increase linearly except for locations near the surface. For depths greater than $12 \mathrm{~mm}, t_{1 / 2}$ and $\langle t\rangle$ remain proportional to the object-detector distance. These results agree well with those reported in other studies $[15,40]$. Furthermore, the increase of the source-detector distance contributes to shift $t_{1 / 2}$ and $\langle t\rangle$ to greater times, but this effect is more pronounced for superficial depth locations than for deep depths. Whatever the source-detector spacing may be, the difference between $\langle t\rangle$ and $t_{1 / 2}$ appears as constant, and consequently cannot be used as an indicator of the inclusion localization. Monte Carlo data and analytical results confirm these different trends and agree quantitatively to within few percent of each other for the three mentioned source-detector separations.

The effect of varying the radius of the spherical fluorescent object located at fixed depth $15 \mathrm{~mm}$ is depicted in Fig. 2(b). It can be seen that the more the object radius, the smaller the time $\left(t_{1 / 2}\right.$ or $\langle t\rangle)$ to reach the surface of the medium. This phenomenon may be explained by the fact that early or late reemitted fluorescent photons are preferentially located at the periphery part of the sphere facing the laser source. Therefore, for a fixed depth of the object center, different diameters lead to different distances between the top of the object and the surface of the surrounding medium, then contributing to vary the two time delays. We also observe an almost linear relationship between both arrival times which is slightly affected (10-15\%) by the biomarker concentration that vary from $0.1 \mu \mathrm{M}$ to $3 \mu \mathrm{M}$. Moreover, the increase of the 

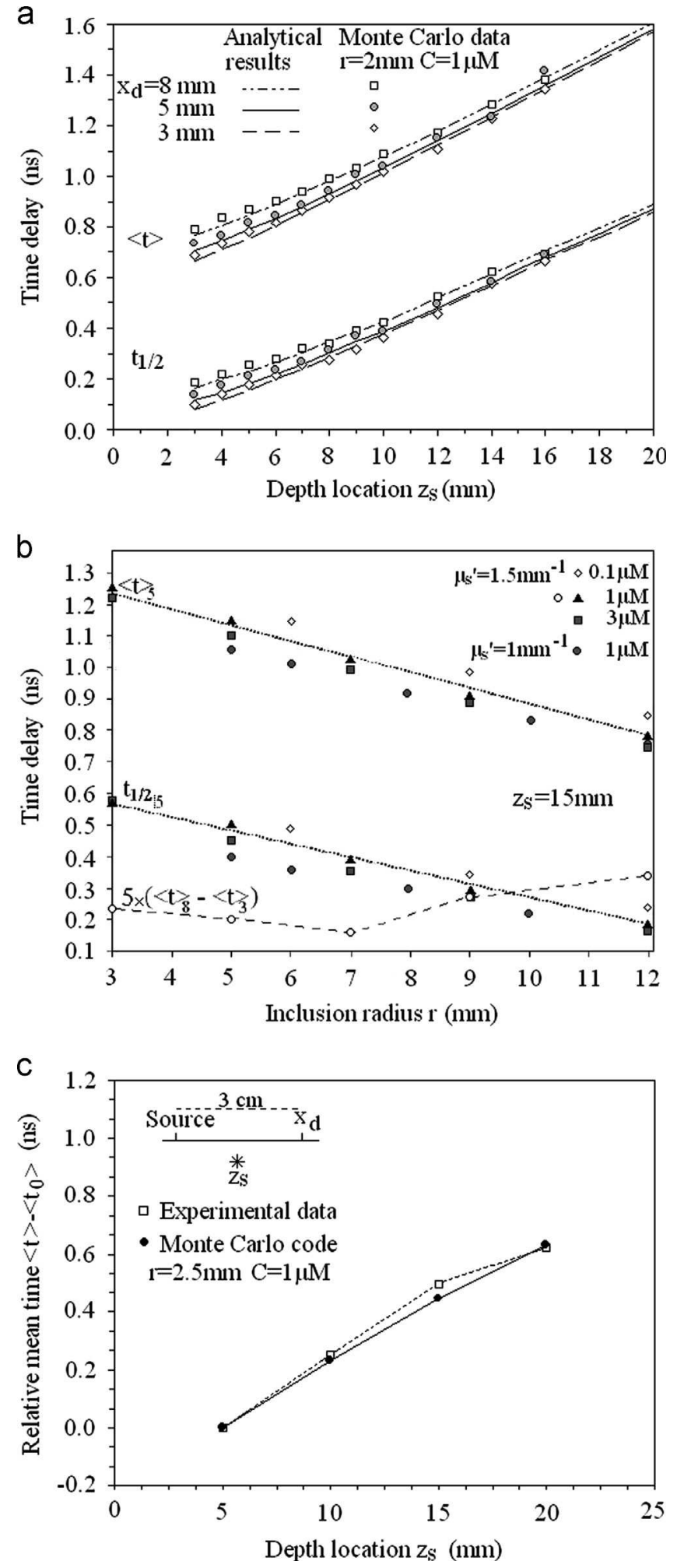

Fig. 2. (a) Comparison of analytical model [11] and Monte Carlo results showing the plots of $t_{1 / 2}$ and $\langle t\rangle$ versus the depth location $z_{\mathrm{s}}$ of a spherical object $(r=2 \mathrm{~mm}, C=1 \mu \mathrm{M} / \mathrm{ICG})$ embedded in a semi-infinite homogeneous medium $\left(\mu_{\mathrm{a}}=0.03 \mathrm{~mm}^{-1}, \mu_{\mathrm{s}}^{\prime}=1.5 \mathrm{~mm}^{-1}\right)$, for three source-detector separations $x_{\mathrm{d}}=3,5$ and $8 \mathrm{~mm}$. (b) Plots of $t_{1 / 2},\langle t\rangle$ and $\Delta\langle t\rangle$ versus the inclusion radius varying from 3 to $12 \mathrm{~mm}$. Monte Carlo results refer to a spherical object with fixed ICG concentration $(0.1-3 \mu \mathrm{M})$ and immersed at $z_{\mathrm{s}}=15 \mathrm{~mm}$, in two different semiinfinite scattering media $\left(\mu_{a}=0.03 \mathrm{~mm}^{-1}, \mu_{s}^{\prime}=1\right.$ or $\left.1.5 \mathrm{~mm}^{-1}\right)$. (c) Comparisons of experimental results [32] and Monte Carlo simulations showing the changes of the time delay $\langle t\rangle-\left\langle t_{0}\right\rangle$ versus the depth location $z_{\mathrm{s}}$ of a fluorescent object $(r=2.5 \mathrm{~mm}$, $C=1 \mu \mathrm{M} / \mathrm{ICG})$ immersed in a scattering liquid medium $\left(\mu_{a}=0.002 \mathrm{~mm}^{-1}\right.$, $\mu_{\mathrm{s}}^{\prime}=1 \mathrm{~mm}^{-1}$ ). reduced scattering of the medium from 1 to $1.5 \mathrm{~mm}^{-1}$ delays the arrival times. These findings qualitatively agree with other published results [41,42]. The difference between two mean-times $\Delta\langle t\rangle$ acquired at $x_{\mathrm{d}}=8 \mathrm{~mm}$ and $3 \mathrm{~mm}$ and plotted against the inclusion radius is also displayed in the Fig. 2(b). A factor equal to 5 was necessary to scale the data together with the plots corresponding to $t_{1 / 2}$ or $\langle t\rangle$. The curve reveals two different parts which depend on the inclusion radius. The difference $\Delta\langle t\rangle$ decreases from 3 to $7 \mathrm{~mm}$, and increases from 7 to $12 \mathrm{~mm}$. This suggests the possibility to detecting a threshold inclusion radius, and also the rate of increase of a tumor based on time-dependent information acquired on the scalp surface. Although the critical radius $(r=7 \mathrm{~mm})$ may be slightly dependent on inclusion concentration $(0.1-3 \mu \mathrm{M})$ and scattering amount of the surrounding medium (results not shown), the method needs to be more deeply investigated.

The changes in the mean-time of arrival photons, $\langle t\rangle$, resulting from different depth positions of a fluorescent spherical object $(r=2.5 \mathrm{~mm}, C=1 \mu \mathrm{M})$ embedded in a diffusive liquid medium $\left(\mu_{a}=0.002 \mathrm{~mm}^{-1}, \mu_{s}^{\prime}=1 \mathrm{~mm}^{-1}\right)$, are compared to experimental results recently reported by Kacprzak et al. [32] in Fig. 2(c). Monte Carlo simulations assume that the object is on the mid-plane between the source and the detector; whereas the results were normalized by the value $\left\langle t_{0}\right\rangle$ determined when the center of the inclusion was positioned $2.5 \mathrm{~mm}$ below the surface. The computations agree well with the experiments, in the range $5-15 \mathrm{~mm}$ and again show that the mean time of arrival of fluorescent photon does not depend strongly on concentration of ICG in the inclusion. When the inclusion is located deeper $\left(z_{\mathrm{s}}>15 \mathrm{~mm}\right)$, the relative time $\langle t\rangle-\left\langle t_{0}\right\rangle$ still increases, but the curves slightly deviate from their initial linear slopes. However, it seems difficult to apply such normalization procedure in clinical routine, because $\left\langle t_{0}\right\rangle$ cannot be easily assessed.

\subsection{Multi-layered structure of the brain}

\subsubsection{Three-layer model}

The following computations based on the Monte Carlo code were firstly related to a simplified three-layer architecture, where the total thickness of the scalp and the skull was reduced to a $4 \mathrm{~mm}$ layer of bone. The CSF layer thickness was held constant at $4 \mathrm{~mm}$, whereas the gray matter was considered as infinite. A fluorescent sphere of radius $3 \mathrm{~mm}$ and containing $1 \mu \mathrm{M}$ of ICG, was located at different depths varying from $7 \mathrm{~mm}$ to $15 \mathrm{~mm}$, and three source-detector separations, $x_{\mathrm{d}}=2,6$ and $8 \mathrm{~mm}$ were considered. The optical properties of the different layers were chosen according to Boas et al. [20]: tissue bone layer $\mu_{\mathrm{a}}=0.04 \mathrm{~mm}^{-1}-\mu_{\mathrm{s}}{ }^{\prime}=1 \mathrm{~mm}^{-1}$, CSF layer $\mu_{\mathrm{a}}=0.004$ $\mathrm{mm}^{-1}-\mu_{\mathrm{s}}{ }^{\prime}=0.25 \mathrm{~mm}^{-1}$, and gray matter $\mu_{\mathrm{a}}=0.025 \mathrm{~mm}^{-1}-\mu_{\mathrm{s}}{ }^{\prime}=$ $1.25 \mathrm{~mm}^{-1}$. To consider different scattering and absorption levels in the CSF layer, the optical properties were altered in the following ranges $\mu_{\mathrm{aCSF}}\left\{0.004-0.04 \mathrm{~mm}^{-1}\right\}$ and $\mu_{\mathrm{s}}^{\prime} \mathrm{CSF}\left\{0.25-1 \mathrm{~mm}^{-1}\right\}$. Fig. 3 shows the plots of the mean-arrival-time $\langle t\rangle$ against the depth $z_{\mathrm{s}}$ (continuous lines). We note that the impact of changing $\mu_{\mathrm{s}}^{\prime}{ }_{\mathrm{CSF}}$ or $\mu_{\mathrm{acSF}}$, on the calculated values was even small for the three investigated source-detector geometries. By another way, the general trend of the three curves is the same as this displayed on the Fig. 2 (a). This contributes to generalize in a way the results obtained in homogeneous media, while reemphasizing the major role that plays the source-detector spacing on the mean-time $\langle t\rangle$. According to Liebert et al. [43], the major problem linked to the determination of the mean-time-of-flight $\langle t\rangle$ lies in the sensitiveness of the result to the noise background and also to the finite integration range. Before examining this question, we note that the convolution of the impulse response data with the decay rate of the fluorescent dye largely contributes to reduce the noise inherent in the M.C. simulations. Consequently, an upper time of integration of about $6.4 \mathrm{~ns}$ and a 


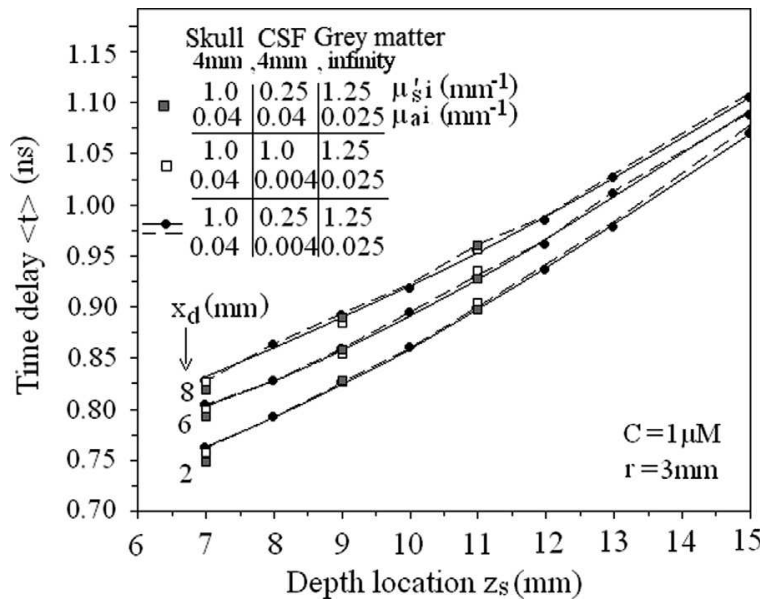

Fig. 3. Plots of the mean-time-of-arrival-photons $\langle t\rangle$ versus the depth location of a diseased region ( $r=3 \mathrm{~mm}, C=1 \mu \mathrm{M} / \mathrm{ICG})$ embedded in a three-layered head model. Monte Carlo simulations refer to three source-detector spacing $x_{\mathrm{d}}=2,6$ and $8 \mathrm{~mm}$, and different combinations of optical parameters $\left(\mu_{s}^{\prime}, \mu_{\mathrm{a}}\right)$ of the CSF layer. The data were generated from time-of-flight curves with (broken lines) or without added noise (continuous lines).

lower time close to zero were used to achieve the results relating to $\langle t\rangle$. Nevertheless, in practice (or in clinical settings), as might be expected, the recorded time of flight curves are much noised especially at earliest and latest times. We then tested the noise robustness of our method by reconsidering the results depicted on the Fig. 3, for $x_{d}=2,6$ and $8 \mathrm{~mm}$, using the following set of thickness and optical properties: skull $/ 4 \mathrm{~mm}, \mu_{a}=0.04 \mathrm{~mm}^{-1}-\mu_{s}{ }^{\prime}=1 \mathrm{~mm}^{-1}$; $\mathrm{CSF} / 4 \mathrm{~mm}, 0.004 \mathrm{~mm}^{-1}-\mu_{s}^{\prime}=0.25 \mathrm{~mm}^{-1}$; gray matter/ as infinite, $\mu_{a}=0.025 \mathrm{~mm}^{-1}-\mu_{s}{ }^{\prime}=1.25 \mathrm{~mm}^{-1}$. Each convolved curve corresponding to the depth location $\left(7 \mathrm{~mm} \leq z_{s} \leq 15 \mathrm{~mm}\right)$ of a fluorescent inclusion ( $r=3 \mathrm{~mm}, C=1 \mu \mathrm{M}$ ) embedded in the three-layer head model were then noised by adding the Poisson noise (mean: 10, standard deviation: 10) to mimic at best experimental time of flight curves. Thereafter, the mean-times $\langle t\rangle$ were recalculated by using a time range of integration $\left\{t_{\text {lower }} \leq t_{\text {limit }} \leq t_{\text {upper }}\right\}$, where the upper and lower times are respectively defined as the times at whom the curves reach $30 \%$ of their maxima and decrease up to $1 \%$ of their maxima. Typical results (broken lines) are shown in Fig. 3. Interestingly, the difference between the data calculated from the consideration of time-of-flight curves with and without noise remains on the uncertainty range of our measurements $(\approx 20 \mathrm{ps})$.

Subsequently, we carried out another moment analysis based on the calculation of the variance (second centralized moment) which is defined as $V=\left\langle t^{2}\right\rangle-\langle t\rangle^{2}[15,43]$. For that purpose, we used the same head tissue arrangement as described before, except that the radius of the fluorescent inclusion $(C=1 \mu \mathrm{M})$ was varied according to three different values: 2,3 , and $5 \mathrm{~mm}$. A systematic comparison between data obtained with $\langle t\rangle$ and $V$ showed that the variance does not take full advantage of recovering the depth or size change of the inclusion. In addition, the variance is more affected by the noise than the mean-time-of-flight, which can be problematic for probing deep diseased location in the brain tissue.

\subsubsection{Five-layer model}

Next, two series of measurements were performed according to the five-layered structure as displayed Fig. 1(a). The total thickness of the upper layers (scalp and skull) was set to $10 \mathrm{~mm}$. The thickness of the scalp was of $4 \mathrm{~mm}$, the one of the gray layer was held constant at $4 \mathrm{~mm}$, the cerebrospinal fluid CSF was of $2 \mathrm{~mm}$ thick, whereas the white matter was considered as infinite. The optical properties of each considered plane layer were adapted from those reported by Okada et al. [21], scalp: $\mu_{\mathrm{a}}=0.018 \mathrm{~mm}^{-1}-\mu_{\mathrm{s}}^{\prime}=1.9 \mathrm{~mm}^{-1}$; $\quad$ skull: $\quad \mu_{\mathrm{a}}=0.016 \mathrm{~mm}^{-1}-\mu_{\mathrm{s}}^{\prime}=$ $1.6 \mathrm{~mm}^{-1}$; gray matter: $\mu_{\mathrm{a}}=0.03 \mathrm{~mm}^{-1}-\mu_{\mathrm{s}}{ }^{\prime}=2.2 \mathrm{~mm}^{-1}$; white matter: $\mu_{\mathrm{a}}=0.014 \mathrm{~mm}^{-1}-\mu_{\mathrm{s}}{ }^{\prime}=9.1 \mathrm{~mm}^{-1}$. The absorption coefficient of the CSF layer was held constant at $0.004 \mathrm{~mm}^{-1}$. However, its reduced scattering coefficient $\mu_{\mathrm{s}}^{\prime}{ }^{\prime} \mathrm{CSF}$ was set to $0.1 \mathrm{~mm}^{-1}$ or considered as varying in the range $0.1-1 \mathrm{~mm}^{-1}$.

3.2.2.1. Modifying the location and shape of the inclusion. In a first series of measurements, the two following situations were considered: (i) the inclusion $(r=3 \mathrm{~mm}, C=1 \mu \mathrm{M}-\mathrm{ICG})$ was moved from $z_{\mathrm{s}}=15 \mathrm{~mm}$ to $17 \mathrm{~mm}$, and (ii) the inclusion located at $z_{\mathrm{s}}=15 \mathrm{~mm}$ had a radius of $5 \mathrm{~mm}$, with a lower concentration $C=0.2 \mu \mathrm{M}$. In each case $\mu_{\mathrm{s}}^{\prime} \mathrm{CSF}=0.1 \mathrm{~mm}^{-1}$ and $x_{d}=5 \mathrm{~mm}$, whereas the computed data were normalized by the value $\left\langle t_{0}\right\rangle$ determined when the inclusion $(r=3 \mathrm{~mm}, C=1 \mu \mathrm{M})$ was located at $z_{s}=15 \mathrm{~mm}$. In the first scenario that corresponds to an increase of the depth location inside the gray matter, a difference $\langle t\rangle-\left\langle t_{0}\right\rangle$ of $+80 \mathrm{ps}$ was noted. In the second that represents a tumor growth (from $3 \mathrm{~mm}$ to $5 \mathrm{~mm}$ ), the difference $\langle t\rangle-\left\langle t_{0}\right\rangle$ was about $-100 \mathrm{ps}$. This temporal shift can be even increased by locating the detector closer to the source. For example, for a source-detector spacing equal to $3 \mathrm{~mm}$ which seems reasonable to avoid tissue auto fluorescence, the absolute time shifts $\left|\langle t\rangle-\left\langle t_{0}\right\rangle\right|$ remain equal to 160 ps. Interestingly, the sign $(+,-)$ allows to distinguish between these two situations. However, according to the Fig. 2(b), this distinction is only possible for an inclusion radius varying up to $7 \mathrm{~mm}$. Nonetheless, these findings are encouraging and show a first step in the noninvasive diagnosis of spatial evolutions of early tumors embedded in complex head models.

To demonstrate the interest of using a simple head model and explore the effects of variability between subjects, we have performed different complementary simulations. The study was carried out in two stages. (i) The thicknesses of the scalp, skull, CSF, gray matter, and white matter were varied, while the optical parameters are fixed, except for $\mu_{\text {scsf }}^{\prime}$ for which three values were respectively assigned: $0.25 \mathrm{~mm}^{-1}, 1 \mathrm{~mm}^{-1}$, and $0.1 \mathrm{~mm}^{-1}$ (See Table 2). (ii) The thicknesses related to the scalp, skull, CSF, gray matter, and white matter were fixed, while the reduced coefficient of each component is varied (See Table 3). All the data were generated with $z_{\mathrm{s}}=15 \mathrm{~mm}, r=3 \mathrm{~mm}, C=1 \mu \mathrm{M}$, and $x_{\mathrm{d}}=5 \mathrm{~mm}$. It is shown that the mean-time-of-flight $\langle t\rangle$ is less dependent on the anatomical head structure than the spatial distribution of scattering coefficients ( $\sim \%$ against $10 \%)$. Furthermore, the results depicted in Table 3 underline the prominent role played by the superficial tissue (scalp and skull) on the estimation of the meantime-of-photon-flight (and then the depth location of the

Table 2

Mean-time-of-flight $\langle t\rangle$, calculated for various anatomical head models. The data, computed from time-of-flight curves with (italic numbers) or without added noise (heavy numbers), were generated with $z_{\mathrm{s}}=15 \mathrm{~mm}, r=3 \mathrm{~mm}, C=1 \mu \mathrm{M}, x_{\mathrm{d}}=5 \mathrm{~mm}$, and are based on fixed optical parameter values for each tissue component.

\begin{tabular}{|c|c|c|c|c|c|c|c|}
\hline \multirow{2}{*}{$\begin{array}{l}\text { Tissue type } \\
\text { Scalp }\end{array}$} & \multirow{2}{*}{$\begin{array}{l}\begin{array}{l}\mu_{a} \\
\left(\mathrm{~mm}^{-1}\right)\end{array} \\
0.018\end{array}$} & \multirow{2}{*}{$\begin{array}{l}\begin{array}{l}\mu_{s}{ }^{\prime} \\
\left(\mathrm{mm}^{-1}\right)\end{array} \\
1.9\end{array}$} & \multicolumn{5}{|c|}{ Thickness (mm) } \\
\hline & & & 3 & 3 & 3 & 3 & 3 \\
\hline Skull & 0.016 & 1.6 & 4 & 5 & 7 & 5 & 5 \\
\hline CSF & 0.004 & $\mu_{s}^{\prime}{ }_{\text {csf }}$ & 2 & 2 & 2 & 1 & 4 \\
\hline Gray matter & 0.036 & 2.2 & 8 & 8 & 8 & 8 & 8 \\
\hline $\begin{array}{l}\text { White } \\
\text { matter }\end{array}$ & 0.014 & 9.1 & infinity & infinity & infinity & infinity & infinity \\
\hline \multirow[t]{4}{*}{$\langle t\rangle(\mathrm{ns})}$, & \multicolumn{2}{|c|}{$\mu_{s \mathrm{csf}}^{\prime}=0.25 \mathrm{~mm}^{-1}$} & $\begin{array}{l}\mathbf{1 . 4 3} \\
1.44\end{array}$ & 1.43 & 1.43 & 1.45 & 1.43 \\
\hline & \multirow{3}{*}{\multicolumn{2}{|c|}{$\begin{array}{l}\mu_{s^{\prime} \mathrm{csf}}^{\prime}=1 \mathrm{~mm}^{-1} \\
\mu_{s^{\prime} \mathrm{csf}}^{\prime}=0.1 \mathrm{~mm}^{-1}\end{array}$}} & & & & 1.45 & 1.45 \\
\hline & & & & 1.45 & & 1.46 & 1.41 \\
\hline & & & & & & 1.48 & 1.42 \\
\hline
\end{tabular}


fluorescent inclusion). This suggests that an initial estimate of the distribution of optical properties is needed, when the depth location of the fluorescent tumor is required. The use of prior information provided by an atlas $[34,44,45]$ can enhance the robustness of such a procedure, while reducing the effects due to inter-subject variations or subject age group. We again note (Tables 2 and 3), that the data (italic numbers) referring to noised time-of-flight curves (Poisson noise, mean: 10, standard deviation: 10) are quite close to those (heavy numbers) obtained without added noise.

In a second series of measurements, the possibility to observe the changes of the shape of the region containing the fluorophores was investigated. As depicted in Fig. 4(a1-c2) the shape of the fluorophores progressively evolves from a spheroid model $\left(a_{1}, b_{1}\right.$, $b_{2}$ ) to an elongated ellipsoid $\left(c_{2}\right)$ directed along the coordinate $x$. Three indicators were used to follow these shape changes: (1) the mean-time-of-flight $\langle t\rangle,-2)$ the second moment $\rho_{\sigma}$ defined as

$\rho_{\sigma}=\sqrt{\left\langle\rho^{2}\right\rangle}=\sqrt{\frac{\iint\left(x^{2}+y^{2}\right) F(x, y) \mathrm{d} x \mathrm{~d} y}{\iint F(x, y) \mathrm{d} x \mathrm{~d} y}}$

and the time-reflected intensity distributions found by scanning the source-detector tandem $\left(x_{\mathrm{d}}=5 \mathrm{~mm}\right)$ along the scalp surface. All the results are listed in the Table 4 . It is shown that the mean

\section{Table 3}

Mean-time-of-flight $\langle t\rangle$, calculated for various spatial distribution of optical parameters in the head model. The data, computed from time-of-flight curves with (italic numbers) or without added noise (heavy numbers), were generated with $z_{\mathrm{s}}=15 \mathrm{~mm}, r=3 \mathrm{~mm}, C=1 \mu \mathrm{M}, x_{\mathrm{d}}=5 \mathrm{~mm}$, and are based on fixed thickness values for each tissue type.

\begin{tabular}{llllllll}
\hline Tissue type & Thickness $(\mathrm{mm})$ & $\mu_{\mathrm{a}}\left(\mathrm{mm}^{-1}\right)$ & \multicolumn{5}{c}{$\mu_{\mathrm{s}}^{\prime}\left(\mathrm{mm}^{-1}\right)$} \\
\hline Scalp & 4 & 0.018 & 1.9 & 1.9 & 1.9 & $1.71 \mathrm{a}$ & $1.27 \mathrm{~b}$ \\
Skull & 6 & 0.016 & 1.6 & 1.6 & 1.6 & $1.44 \mathrm{a}$ & $1.07 \mathrm{~b}$ \\
CSF & 2 & 0.004 & 0.1 & 0.25 & 1.6 & 0.25 & 0.25 \\
Gray matter & 4 & 0.036 & 2.2 & 2.2 & 2.2 & 2.2 & 2.2 \\
White matter & infinity & 0.014 & 9.1 & 9.1 & 9.1 & 9.1 & 9.1 \\
$\langle t\rangle(\mathrm{ns})$ & & & $\mathbf{1 . 4 6}$ & $\mathbf{1 . 4 7}$ & $\mathbf{1 . 4 8}$ & $\mathbf{1 . 4 2}$ & $\mathbf{1 . 3 5}$ \\
& & & 1.47 & & & & \\
\hline
\end{tabular}

a Data based on $90 \%$ of $\mu_{s}^{\prime}$ related to Scalp and Skull.

${ }^{\mathrm{b}}$ Data based on $67 \%$ of $\mu_{s}^{\prime}$ related to Scalp and Skull. time $\langle t\rangle$ is a relevant parameter to characterize the cases $a_{1}, b_{1}$, and $b_{2}$, where the centroid of the fluorophores is respectively equal to 14,15 , and $17 \mathrm{~mm}$, but not adapted to relate the fluorophore side spreading along the direction $x$ (cases $c_{1}$ and $c_{2}$ ). Moreover the use of the second moment $\langle\rho\rangle$ shows that the inclusion size is $50-60 \%$ larger than the true value in the cases $a_{1}, b_{1}$, and $b_{2}$, but is underestimated in the cases $c_{1}$ and $c_{2}$. These conclusions are independent whether the beam is enlarged $\left(d_{\mathrm{s}}=10 \mathrm{~mm}\right)$ or not $\left(d_{\mathrm{s}} \rightarrow 0\right.$, like a point source). These results still confirm that the diffusion process strongly contributes to enlarge the reconstructed size of objects having a spherical or cylindrical shape, from FWHM measurements [46]. In point of fact, no improvement was observed in the estimation of the lateral spreading of the fluorophore distribution when the laser source was held fixed closest to the axis $\left(x_{s}=0\right)$ of the object. As the fluorescent inclusion laterally extends, only a volume of fluorophores located around the axis of the source remains excited, thus generating fluorescent remitted intensity profiles that give practically the same value of $\langle\rho\rangle$. It is reasonable to expect that measurements performed by using source-detector scanning arrangements will improve the ability to characterize the spreading size along the direction $x$. This is well confirmed in Table 4, where it is shown that scanning together the source-detector $\left(x_{\mathrm{d}}=5 \mathrm{~mm}\right)$ along the scalp surface gives satisfactory results, although the time-gating mode may be more accurate than the continuous mode. Therefore, combining temporal $(\langle t\rangle)$ and spatial (scanning mode) measurements thus enhances the characterization of fluorophore distribution as well as provides a means of distinguishing depth location and lateral extension of fluorescence inside a complex head model.

3.2.2.2. Probing optical changes of the CSF layer. Fig. 5 Another aspect that was considered in this study is the one related to the changes of the scattering properties of the cerebrospinal fluid. A light channeling effect inside a low scattering CSF layer, that could reduce the penetration in deeper domain, has been suggested by few groups [33]. Moreover, an increase of the scattering amount of the CSF layer is well correlated with an increase of the fluid density. This material change will also occur in case of brain injuries, owing to proteins and blood products embedded in the CSF [47]. To probe these changes, we again use the difference $\Delta\langle t\rangle$ computed for two different source-detector

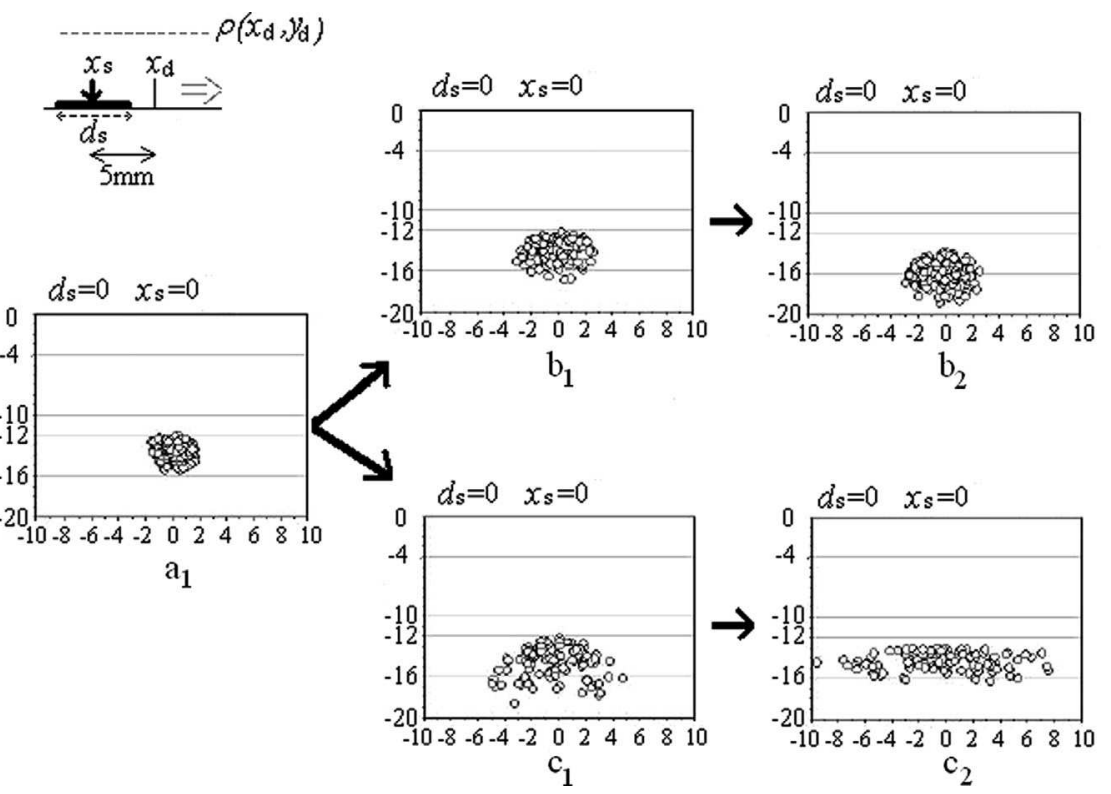

Fig. 4. Sketch of the spatial evolution of fluorescent markers inside a five-layered head model associated to three tested indicators to noninvasively delineate the lateral boundaries. 
Table 4

Results obtained by using the three tested indicators (mean-time-of-flight $\langle t\rangle$, second moment $\rho_{\sigma}$, and scan-profiles resulting from time-gating mode) on the characterization of the shape changes of a fluorescent region embedded in a five-layered head model. (See Fig. 3 for corresponding displays $a_{1}, b_{1}, b_{2}, c_{1}$, and $c_{2}$.)

\begin{tabular}{|c|c|c|c|c|c|c|}
\hline$x_{s}, d_{s}(\mathrm{~mm})$ & Indicator & $a_{1}$ & $b_{1}$ & $b_{2}$ & $c_{1}$ & $c_{2}$ \\
\hline \multirow[t]{4}{*}{$x_{s}=0, d_{s}=0$} & $\langle t\rangle_{x_{\mathrm{d}}}(\mathrm{ns})$ & 1.40 & 1.47 & 1.55 & 1.50 & 1.50 \\
\hline & & $14^{\mathrm{a}}$ & $15^{\mathrm{a}}$ & $17^{\mathrm{a}}$ & $15^{\mathrm{a}}$ & $15^{\mathrm{a}}$ \\
\hline & $\rho=\sqrt{ }\left\langle\rho^{2}\right\rangle(\mathrm{mm})$ & 8.94 & 9 & 9.59 & 9.15 & 10.35 \\
\hline & & $4^{\mathrm{b}}$ & $6^{\mathrm{b}}$ & $6^{\mathrm{b}}$ & $10^{\mathrm{b}}$ & $20^{\mathrm{b}}$ \\
\hline \multirow[t]{2}{*}{$x_{s}=0, d_{s}=10$} & $\langle t\rangle_{X_{\mathrm{d}}}$ (ns) & 1.46 & & 1.56 & & 1.51 \\
\hline & $\rho_{\sigma}=\sqrt{ }\left\langle\rho^{2}\right\rangle(\mathrm{mm})$ & 8.71 & & 9.35 & & 10.62 \\
\hline \multirow[t]{2}{*}{ Scanning $x_{\mathrm{s}}:[-20,0], d_{\mathrm{s}}=0$} & $2\left|X_{\mathrm{d} \text { Threshold } 10 \%}\right|(\mathrm{mm})$ Continuous & 9 & & 13 & & 24 \\
\hline & $2\left|X_{\mathrm{d} \text { Threshold } 10 \%}\right|(\mathrm{mm})$ Time-gating $0<t<1 \mathrm{~ns}$ & 8 & & 13 & & 19 \\
\hline
\end{tabular}

${ }^{\text {a }}$ Depth location $z_{s}$

b True extend along the direction $x$.

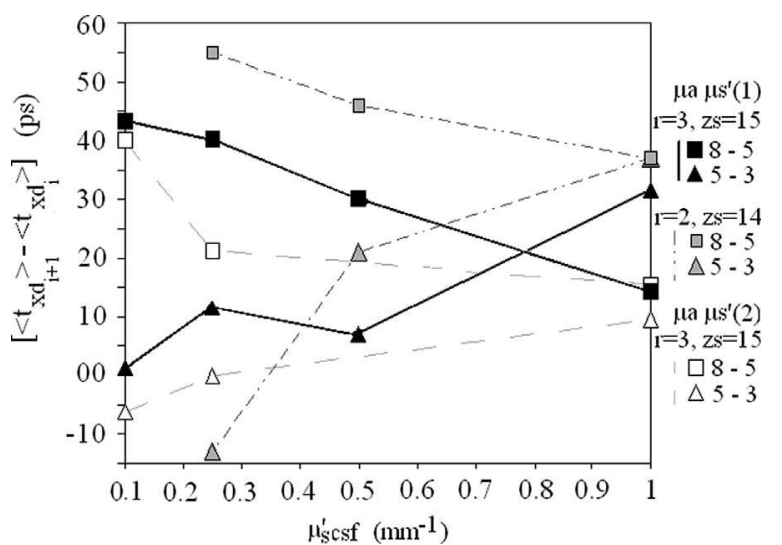

Fig. 5. Plots of $\Delta\langle t\rangle$ acquired at two source-detector separations sets $x_{\mathrm{d}}=5-3 \mathrm{~mm}$, $x_{\mathrm{d}}=8-5 \mathrm{~mm}$, against the change of the reduced scattering coefficient $\left(0.1-1 \mathrm{~mm}^{-1}\right)$ of the CSF layer located in a five-layered head model.

spacing sets: $x_{\mathrm{d}}=8-5 \mathrm{~mm}$, and $x_{\mathrm{d}}=5-3 \mathrm{~mm}$. The Fig. 5 shows the plots of $\Delta\langle t\rangle$ against $\mu_{\mathrm{s}}^{\prime}{ }_{\mathrm{CSF}}$, for $\mu_{\mathrm{s}}{ }_{\mathrm{CSF}}$ ranging from 0.1 to $1 \mathrm{~mm}^{-1}$. It is shown that the difference $\Delta\langle t\rangle$ acquired at $x_{\mathrm{d}}=8$ and $5 \mathrm{~mm}$ decreases as the scattering of the CSF layer increases. In contrast, $\Delta$ $\langle t\rangle$ acquired at $x_{\mathrm{d}}=5$ and $3 \mathrm{~mm}$ shows an increasing trend against $\mu_{\mathrm{s}}^{\prime}$ CSF. Globally, such differences can be easily recorded by using data provided by time-correlated systems. However, the method is not very accurate to uncouple the effects due to a tumor growth or a change of its location, from those related to scattering changes of the CSF layer. Our method is based on the presence of a native embedded tumor in a brain tissue model. If that is not the case, we suggest to inject a fluorescent bolus nearness (or inside) the CSF layer which acts as a "beacon", in order to probe significant scattering alterations occurring in the CSF.

\section{Conclusion}

In this paper, we have succinctly described a computer code based on the Monte Carlo method for investigating timedependent photon migration through multi-layered media that mimic human brain tissue structures bearing a small fluorescent inclusion. By assigning the same optical parameters of each tissue component, the code was first validated against a well known analytical solution, and cross-validated for reported experiments on liquid phantoms containing a spherical fluorescent inclusion. Results of computations show that the time-resolved method may allow the evaluation of depth of fluorescent inclusions. In particular, simulations performed in either homogeneous media or multi-layered media confirm previously reported effect of increase of the mean time of arrival of fluorescence photons originating from deeper regions. Interestingly, this effect depends weakly on the fluorochrome concentration in the inclusion. This seems advantageous for diagnostic purpose for which the assessment of depth location of fluorescent regions is expected, without requiring a specified dye concentration, at each sequence of observation. In a second series of simulations we tried to show that the difference between two mean times acquired at two sourcedetector distances can be used to monitor the inclusion growth or to probe the scattering changes in the CSF layer. This might lead to improved characterization of diseases that can occur in the complex human head. However, the method proposed cannot yet uncouple the two mentioned effects. We also tested the noise robustness of our method, thus showing the possibility to prevent unwanted noise. This feature will greatly enhance the utility of the time-of-flight measurements in a clinical environment. Finally, we showed the possibility to combine time-resolved results with spatial measurements to estimate the shape evolution of a fluorescent region inside a brain tissue (five-layered tissue model). Globally, our results are encouraging and in line with new investigations of the brain tissue devoted to the diagnostic of intracranial targeted tumors.

Although the Monte Carlo method may be considered as a reference in computed optical imaging (or spectroscopy), the above mentioned findings require a confrontation with specific experiments. Future in vitro studies, based on multi-layered phantoms containing small activated fluorescent regions and using time-correlated single-photon counting systems, are then needed to validate the usefulness of our methods. Next paper will be connected with these suggested investigations.

\section{References}

[1] M. Anidiar, O. Cussenot, S. Avrillier, et al., Journal Biomedical Optics 1 (1996) 335.

[2] G. Bourg-Heckly, J. Blais, J.J. Padilla, et al., Endoscopy 32 (2000) 756.

[3] B.C. Wilson, E.M. Sevick, M.S. Patterson, B. Chance, Proceedings of SPIE 80 (1992) 918.

[4] A.P. Gibson, J.P. Hebden, S.R. Arridge, Physics in Medicines and Biology 50 (2005) R1.

[5] A.P. Gibson, H. Dehghani, Philosophical Transactions on Royal Society A 367 (2009) 3055.

[6] E.M. Sevick-Muraca, E. Kuwana, A. Godavarty, et al., Near-infrared fluorescence imaging and spectroscopy in random media and tissues, in: T. Vo Dinh (Ed.), Biomedical Photonics Handbook, CRC Press, 2003.

[7] M.A. Mycek, B.W. Pogue (Eds.), Handbook of Biomedical fluorescence. CRC Press 2003.

[8] V. Ntziachristos, J. Ripoll, R. Weissleider, Optics Letters 27 (2002) 333.

[9] J.L. Kovar, M.A. Simpson, A. Schutz-Geschwander, D.M. Olive, Analytical Biochemistry 361 (2007) 1 . 
[10] J. Wu, L. Perelman, R.R. Dasari, M.S. Feld, Proceedings of National Academy of Sciences United States of America 94 (1997) 8783.

[11] D. Hattery, V. Chernomordik, M. Loew, I. Gannot, A. Gandjbakhche, Journal of the Optical Society of America 18 (2001) 1523.

[12] D. Hall, G. Ma, F. Lesage, Y. Wang, Optics Letters 29 (2004) 2258.

[13] C. D'Andrea, L. Spinelli, A. Bassi, et al., Optics Express 14 (2006) 1888.

[14] J.P. L'Huillier, F. Vaudelle, Optics Express 14 (2006) 12915-12929.

[15] A. Laidevant, A. Da Silva, M. Berger, et al., Applied Optics 46 (11) (2007) 2131.

[16] J. Swartling, D. Bengtsson, K. Terike, et al., Proceedings of SPIE 5693 (2005) 225.

[17] S.J. Erickson, J. Ge, A. Sanchez, A. Godavarty, Translational Oncology 3 (2010) 16.

[18] A. Laidevant, L. Hervé, M. Debourdeau, et al., Biomedical Optics Express 2 (2010) 194.

[19] A. Da Silva, N. Djaker, N. Ducros, et al., Optics Express 18 (2009) 7753.

[20] D.A. Boas, J. Culver, J. Stott, A. Dunn, Optics Express 10 (2002) 159

[21] E. Okada, D.T. Delpy, Applied Optics 42 (2003) 2915.

[22] Y. Fukui, Y. Ajichi, E. Okada, Applied Optics 42 (2003) 2881.

[23] T. Hayashi, Y. Kashio, E. Okada, Applied Optics 42 (2003) 2888.

[24] C. Mansouri, J.P. L'Huillier, N. Kashou, A. Humeau, Lasers in Medical Sciences 25 (2010) 431

[25] A. Liebert, H. Wabnitz, H. Obrig, et al., Neuroimage 31 (2006) 600.

[26] J. Steinbrink, A. Liebert, H. Wabnitz, et al., Neurodegenerative Diseases 5 (2008) 296.

[27] E.M.C. Hillman, Journal Biomedical Optics 12 (051402) (2007) 1.

[28] Y. Hoshi, Y. Shimada, C. Sato, Y. Iquihi, Journal of Biomedical Optics 10 (2005) 064032
[29] H. Zhou, K. Luby-Phelps, B.E. Mickey, et al., Plos One 4 (2009) 1.

[30] B. Tomanek, U. Iqbal, B. Blasiak, et al., Neuro-Oncology 14 (2012) 53.

[31] L. Gagnon, M. Desjardins, J. Jehanne-Lacasse, et al., Optics Express 16 (2008) 15514.

[32] M. Kacprzak, A. Liebert, P. Liebert, et al., Opto-Electronics Review 18 (2010) 37.

[33] A. Custo, W.M. Wells, A. Barnett, et al., Applied Optics 45 (2006) 4747.

[34] F.B. Haeussinger, S. Heinzel, T. Hahn, et al., Plos One 6 (2011) 1

35] A. Liebert, H. Wabnitz, N. Zolek, R. Macdonald, Optics Express 16 (2008) 13188

[36] D.R. Guyer, C.P. Puliafito, J.M. Mones, et al., Ophthalmology 199 (1992) 287.

[37] H.R. Eggert, V. Blazek, Neurosurgery 21 (1987) 459.

[38] L. Wang, S. Jacques, L. Zheng, Computer Methods and Programs in Biomedicine 47 (1995) 131.

[39] J. Swartling, A. Pifferi, A.M.K. Enejder, S. Andersson-Engels, Journal of the Optical Society of America A 20 (2003) 714.

[40] M. Kervella, A. Humeau, J.P. L'Huillier, Ingénierie et Recherche Biomedicale 29 (2008) 20.

[41] M. Kervella, A. Humeau, J.P. L'Huillier, Optics Communications 281 (2008) 5982.

[42] S.H. Han, S. Farshchi-Heydari, D.J. Hall, Biophysical Journal 98 (2010) 350.

[43] A. Liebert, H. Wabnitz, D. Grosenick, et al., Applied Optics 42 (2003) 5785.

44] J. Heiskala, M. Pollari, M. Metsaranta, et al., Optics Express 17 (2009) 14977.

[45] J.C. Mazziotta, A.W. Toga, A. Evans, et al., Neuroimage 2 (1995) 89.

[46] V. Ntziachristos, X.H. Ma, B. Chance, Review of Scientific Instruments 69 (1998) 4221.

[47] D.A. Seehusen, M.M. Reeves, D.A. Fomin, American Family Physician 68 (2003) 1103. 